\title{
Application of the MATLAB Computational Tool for the Problem of the Economic Dispatch of Electric Energy using Genetic Algorithm: Case Study
}

\section{Adriano Lima Pedrosa ${ }^{1}$, Nadime Mustafá Moraes ${ }^{2}$, Jorge Laureano Moya Rodriguez ${ }^{3}$, Manoel Henrique Reis do Nascimento ${ }^{4}$, Silas Farias Fernandes Filho 5}

\footnotetext{
1,5 Aluno do Projeto de Produtividade da Universidade do Estado do Amazonas (UEA).

${ }^{2}$ Orientadora do Projeto de Produtividade da Universidade do Estado do Amazonas (UEA).

${ }^{3}$ Co-orientador do Projeto de Produtividade da Universidade do Estado do Amazonas (UEA).

${ }^{4}$ Profess or do Centro Universitário do Norte (UNINORTE).
}

E-mail: adrianolp01@gmail.com, mustafnadime@gmail.com, jorgemoyar@gmail.com

Received: Ja nuary $13^{\text {th }}, 2017$

Accepted: May $24^{\text {th }}, 2017$

Published: June $30^{\text {th }}, 2017$

Copyright $(02016$ by authors and Institute of Technology Galileo of Amazon (ITEGAM) This work is licensed under the Creative Commons Attribution International License (CC BY 4.0).

http://creativecommons.org/lic enses/by/4.0/

\section{(c) (i) (2) (2) Open Aeces:}

\begin{abstract}
Nowadays, the Brazilian electric sector still has a vast complication in the generation of energy, It has been impacted by some changes that strongly influence this distribution in the energy sector. The economic load dispatch (ELD) comes to assist and supply the demand of the sector in front of some factors that directly interfere in the decentralization of this generation, in the independent production of the conces sionaires and in the intensive use of alternative energy sources.

The objective of the economic load dis patch (ELD) is to decide the power generated by each one of the motors of a thermal plant ensuring that the total cost of the fuel is minimum. At the same time is necessary to guarantee that the total demand and losses at any instant must be equal to the total generation or power supplied by the plant. This paper presents a mathematical model and its solution using the MATLAB optimization toolbox, specifically the genetic algorithms, to solve the ELD problem. In addition, a case study is presented for 10 generating units considering the power losses and the restrictions of the generators, and turning off the less efficient generators.

Keywords: Electric sector, economic dispatch, MATLAB, genetic algorithm.

\section{Aplicação da Ferramenta Computacional MATLAB para o Problema do Despacho Econômico de Energia Elétrica usando Algoritmo Genético: Estudo de caso}

\begin{abstract}
RESUMO
Atualmente, o setor elétrico brasileiro ainda possui uma vasta complicação na geração de energia, tem sido impactado por algumas mudanças que influenciam fortemente essa distribuição no setor energético. O despacho econômico (ELD) vem auxiliar e suprir a demanda do setor frente alguns fatores que interferem diretamente na descentralização dessa geração, na produção independente das concessionárias e no uso intensivo de fontes alternativas. O objetivo do despacho econômico (ELD) é decidir potência gerada por todos e cada um dos motores de uma usina térmica garantindo que o custo total do combustível seja mínimo e, ao mesmo tempo, a demanda e as perdas totais em qualquer instante deve ser igual à geração total ou potência fornecida pela usina. Este artigo apresenta um modelo matemático e a solução usando o toolbox de otimização do MATLAB, especificamente os algoritmos genéticos, para resolver o problema ELD. Além disso é apresentado um estudo de caso para 10 unidades geradoras considerando as perdas de potência e as restrições dos geradores, e desligando os geradores menos eficientes.
\end{abstract}

Palavras Chaves: setor elétrico, despacho econômico, MATLAB, algoritmo genético.

\section{INTRODUÇÃO}

No cenário mundial os desenvolvimentos dos sistemas de energia elétrica representam fundamentalmente a prospecção de novos rumos e planejamentos na área. Em virtude da desregulamentação do setor elétrico brasileiro, problemas recorrentes surgiram ao longo dos tempos quanto suas operacionalidades, provenientes de situações técnicas, ambientais, 
sociais e econômicas. O surgimento de termoelétricas foi fundamental para atender estrategicamente com vantagens peculiares a demanda de energia de forma contínua, principalmente ao que se refere ao retorno dos capitais iniciais investidos emcurto espaço de tempo, além da diversificação da matriz energética. Ademais, é fundamental destacar as desvantagens desse negócio, pois os custos são elevados na produção de energia.

\section{REVISÃO DA LITERATURA}

\section{II.1 DESCRIÇÃO DO PROBLEMA}

O planejamento e operação adequados de um sistema de energia elétrica representam um compromis so entre a minimização dos custos de investimentos e operação e o atendimento a padrões de qualidade da energia final entregue ao consumidor, previamente estabelecidos [1].

Nessa perspectiva de complexidade dos setores envolvidos, sempre encontramos falhas na capacidade de distribuição e demais fatores que apontam e propiciam um estudo colaborativo na produção dessa energia a custos menos elevados e por is so há uma crescente real necessidade de expansão para suprir projetos futuros nesse sentido. Os principais fatores que colaboram para um aumento substancial ao não atendimento das reais necessidades no presente contexto referem-se à deficiência de demanda não atendida em sua completude por parte dos setores.

\section{II.2 DESPACHO ECONÔMICO}

Os custos do combustível e da eficiência da estação de energia determinam os custos operacionais de produção de energia elétrica. Assim, o problema de despacho econômico (DE) tornouse uma tarefa muito importante na operação e planejamento de sistemas de energia. Seu principal objetivo é otimizar a geração de eletricidade a partir das unidades disponíveis, de modo que o custo total de geração é minimizado enquanto as restrições consideradas pelo sistema são satisfeitas [2].

O DE tem como meta estabelecer a alocação ótima da demanda de energia para as unidades geradoras de uma usina termoelétrica, de tal forma que satisfaça os limites operacionais desse sistema com um custo mínimo possível portanto o objetivo básico do problema de DE dos sistemas de energia elétrica (SEE) é minimizar o custo de combustível utilizado na geração de eletricidade [3].

Matematicamente o tipo de problema DE, abordado neste artigo, pode ser descrito como uma função objetivo e duas restrições. As restrições representadas pelas equações (1) e (2) devem ser satisfeitas,

$$
\begin{aligned}
& \sum_{i=1}^{n} P_{i}-P_{L}-P_{D}=0 \\
& P_{i}^{\text {min }} \leq P_{i} \leq P_{i}^{\max }
\end{aligned}
$$

A equação (1) representa as restrições de igualdade do balanço de potência (is to é, balanço entre suprimento e demanda), enquanto a expressão (2) representa as restrições de desigualdade relativas aos limites da capacidade de geração de potência de cada unidade geradora, onde $P_{i}$ é a saída para a unidade geradora $i$ (em $\mathrm{MW}) ; n$ é o número de geradores presente no sistema; $P_{D}$ é a demanda de carga total (em MW); $P_{L}$ são as perdas de transmissão (em MW) e $P_{i}^{\min } e P_{i}^{\max }$ são respectivamente as saídas de operação mínimas e máximas da unidade geradora $i$ (em MW). O custo total de combustível deve ser minimizado conforme representado na equação (3),

$$
\min f=\sum_{i=1}^{n} F_{i}\left(P_{i}\right)
$$

onde $F_{i}$ é a função custo de combustível para a unidade geradora $i(\mathrm{em} \$ / \mathrm{h})$, que é definida pela equação,

$$
F_{i}\left(P_{i}\right)=a_{i} P_{i}^{2}+b_{i} P_{i}+c_{i}
$$

onde $a_{i}, b_{i}$ e $c_{i}$ são coeficientes de custo do gerador $i$.

Ao longo dos últimos anos, têm sido propostos vários métodos para solucionar o problema de ED. Eles podem ser divididos em três grupos principais: Clássico, baseado em Inteligência Artificial (AI) e Métodos Híbridos [2].

\section{II.3 ALGORITMOS GENÉTICOS}

Os algoritmos genéticos (AG), criado por Holland, são uma classe particular de algoritmos evolutivos que usam técnicas inspiradas pela biologia evolutiva [4].

Tentativas de pesquis adores de todo o mundo, para superar as limitações da programação matemática convencional, são conduzidas para introduzir algoritmos meta-heurísticos como algoritmo genético (AG), recozimento simulado (SA), programação evolucionária (EP) e método hierárquico e outros,que demonstraram ser muito eficientes na resolução de problemas complexos do sistema elétrico de potência, contudo, estes métodos heurísticos não garantem sempre a solução globalmente ideal [5].

A partir da década de 50, métodos heurísticos foram propostos para simular fenômenos biológicos, entre estes, os Algoritmos Genéticos (AG’s). Este método se baseia no processo de seleção natural e evolução das espécies. Na analogia matemática, os indivíduos da população são os pontos de partida ou soluções iniciais. É importante destacar, que os AG's trabalham sempre com um conjunto de indivíduos. A determinação do número de indivíduos é um parâmetro que deve ser ajustado para cada caso e não existe um parâmetro ótimo, porém, há como se determinar uma faixa de trabalho [6].

Os operadores básicos dos AG’s são a criação da população inicial aleatoriamente, o cruzamento, a mutação e a seleção. Inicialmente, a população inicial possui indivíduos de características boas e ruins, devido ao processo de criação que é aleatório. Estas características podem ser trocadas com outros indivíduos da população e transmitidas para as gerações posteriores através do operador cruzamento.

No operador mutação, os indivíduos são "modificados" na tentativa de torná-los mais aptos a permanecerem nas próximas gerações. Na analogia matemática, a aptidão está relacionada à função objetivo. No operador seleção, os indivíduos mais aptos serão escolhidos para fazerem parte das gerações posteriores [7]. 


\section{II.4 MATLAB OU SCILAB}

O Scilab (Scientific Laboratory) é um ambiente gráfico para cálculo científico disponível gratuitamente desde 1994 e desenvolvido em 1990 por pesquisadores do "Institut Nationale de Rechercheen Informatique ET em Antomatique - (INRIA)" e "École Nationale dês Ponts et Chaussée - (ENPC)" na França [8] e [9].

Tem sido amplamente utilizado em muitas Universidades e empresas ao redor do mundo para processamento de imagem, computação, modelagem, oferecendo habilidade na construção de programas voltados para as diversas áreas do conhecimento científico.

MATLAB é uma linguagem de alto desempenho para computação técnica. Ele integra computação, visualização e programação em um ambiente fácil de usar onde os problemas e soluções são expressos em notação matemática familiar. Os usos típicos incluem:

\section{$\checkmark \quad$ Matemática e computação \\ $\checkmark$ Desenvolvimento de Algoritmos \\ $\checkmark$ Modelagem, simulação e prototipagem \\ $\checkmark$ Análise, exploração e visualização de dados \\ $\checkmark$ Gráficos científicos e de engenharia}

$\checkmark$ Desenvolvimento de aplicativos, incluindo criação de interfaces gráficas de usuário

MATLAB é um sis tema interativo cujo elemento de dados básico é um array que não requer dimensionamento. Isso permite que muitos problemas de computação técnica sejam resolvidos facilmente, especialmente aqueles com formulações de matriz e vetor, em uma fração do tempo que levaria para escrever um programa em uma linguagem escalar não-interativa como $\mathrm{C}$ ou Fortran.

O nome MATLAB significa laboratório matricial. MATLAB foi escrito originalmente para fornecer acesso fácil ao software matricial desenvolvido pelos projetos LINPACK e EISPACK, que juntos representam o estado da arte em software para computação matricial [10].

MATLAB evoluiu ao longo de um período de anos com a entrada de muitos usuários. Em ambientes universitários, é a ferramenta de instrução padrão para cursos introdutórios e avançados em matemática, engenharia e ciência. Na indústria, MATLAB é a ferramenta de escolha para pesquisa, desenvolvimento e análise de alta produtividade [11]. O MATLAB possui uma família de soluções específicas de aplicativos, chamadas caixas de ferramentas. Muito importante para a maioria dos usuários do MATLAB, toolboxes permitem que você aprenda e aplique tecnologia especializada. As caixas de ferramentas são coleções abrangentes de funções MATLAB (M-files) que estendem o ambiente MATLAB para resolver classes particulares de problemas. Áreas nas quais as caixas de ferramentas estão disponíveis incluemprocessamento de sinais, sistemas de controle, redes neurais, lógica fuzzy, wavelets, simulação e muitos outros [12].

O Matlab e o Scilab são excelentes ferramentas de suporte para as linhas de pesquis a onde o uso de computadores na res olução numérica de problemas é intensivo [13].

O Scilab atende à maioria das necessidades dos cursos de matemática e de seus alunos, porém o Matlab, como era de se esperar (há uma razão para ele ser pago!), é mais eficiente, abrangente, tem recursos para cálculos mais avançados e também é mais conhecido no meio matemático e das engenharias [14][15].

\section{MATERIAIS E MÉTODOS}

\section{III.1 FONTE E COLETA DE DADOS}

A fonte e coleta de dados serão de uma usina do Polo Industrial de Manaus, serão realizadas simulações em ambiente MATLAB utilizando um sistema de teste com 10 geradores, o sistema teste será resolvido pelo Algoritmo Genético e os resultados mostrarão sua eficiência através das simulações para alocação do ótimo no despacho econômico.

\section{III.2 APRESENTAÇÃO DOS DADOS COLETADOS COM RESPECTIVA FONTE}

A Planta de referência selecionada para o estudo de caso é composta de 10 motores, como mostrado na Tabela 1.

Tabela 1: Dados característicos dos motores da planta

\begin{tabular}{|l|c|c|c|c|c|}
\hline Motor & $\begin{array}{c}a_{i} \\
\left(\$ / \mathrm{mW}^{\wedge}\right)\end{array}$ & $\begin{array}{c}b_{i} \\
(\$ / \mathrm{mW})\end{array}$ & $\begin{array}{c}c_{i} \\
(\$)\end{array}$ & $\begin{array}{c}P_{\min } \\
(\mathrm{mW})\end{array}$ & $\begin{array}{c}P_{\max } \\
(\mathrm{mW})\end{array}$ \\
\hline 1. & 0.007 & 7 & 240 & 0.66 & 3.35 \\
\hline 2. & 0.0095 & 10 & 200 & 0.9 & 3.7 \\
\hline 3. & 0.009 & 8.5 & 220 & 0.8 & 3.6 \\
\hline 4. & 0.009 & 11 & 200 & 0.66 & 3.35 \\
\hline 5. & 0.008 & 10.5 & 220 & 0.72 & 3.45 \\
\hline 6. & 0.0075 & 12 & 120 & 0.66 & 2.97 \\
\hline 7. & 0.0075 & 14 & 130 & 0.88 & 3.5 \\
\hline 8. & 0.0075 & 14 & 130 & 0.754 & 3.33 \\
\hline 9. & 0.0075 & 14 & 130 & 0.9 & 3.9 \\
\hline 10. & 0.0075 & 14 & 130 & 0.56 & 2.35 \\
\hline
\end{tabular}

Fonte: Autores, (2107).

Os coeficientes de perdas $\left(B_{m}\right)$ são dados por uma matriz quadrada de tamanho $n \times n$, onde $n$ é o número de geradores (ver tabela 2).

Tabela 2: Matriz de perdas dos 10 motores da usina (todos os valores devem multiplicar-se por 1e-4).

\begin{tabular}{|l|l|l|l|l|l|l|l|l|l|l|}
\hline $\mathrm{M}$ & 1 & 2 & 3 & 4 & 5 & 6 & 7 & 8 & 9 & 10 \\
\hline 1 & 0.14 & 0.17 & 0.15 & 0.19 & 0.26 & 0.22 & 0.34 & 0.38 & 0.43 & 0.45 \\
\hline 2 & 0.17 & 0.6 & 0.13 & 0.16 & 0.15 & 0.2 & 0.23 & 0.56 & 0.23 & 0.51 \\
\hline 3 & 0.15 & 0.13 & 0.65 & 0.17 & 0.24 & 0.19 & 0.25 & 0.38 & 0.43 & 0.45 \\
\hline 4 & 0.19 & 0.16 & 0.17 & 0.71 & 0.3 & 0.25 & 0.43 & 0.56 & 0.23 & 0.51 \\
\hline 5 & 0.26 & 0.15 & 0.24 & 0.3 & 0.69 & 0.32 & 0.18 & 0.37 & 0.42 & 0.48 \\
\hline 6 & 0.22 & 0.2 & 0.19 & 0.25 & 0.32 & 0.85 & 0.97 & 0.55 & 0.27 & 0.58 \\
\hline 7 & 0.22 & 0.2 & 0.19 & 0.25 & 0.32 & 0.85 & 0.67 & 0.38 & 0.43 & 0.45 \\
\hline 8 & 0.19 & 0.7 & 0.13 & 0.18 & 0.16 & 0.21 & 0.28 & 0.56 & 0.23 & 0.51 \\
\hline 9 & 0.26 & 0.15 & 0.24 & 0.3 & 0.69 & 0.32 & 0.18 & 0.37 & 0.42 & 0.48 \\
\hline 10 & 0.15 & 0.13 & 0.65 & 0.17 & 0.24 & 0.19 & 0.25 & 0.38 & 0.43 & 0.45 \\
\hline
\end{tabular}

Fonte: Autores, (2107). 


\section{III.3 MODELO MATEMÁTICO}

O modelo matemático está composto essencialmente pela função do custo (Eq.4) a qual deve ser minimizada (Eq.3). Serão usados os Algoritmos Genéticos com as seguintes características:

$\checkmark$ População: 300 indivíduos.

$\checkmark$ Gerações: 1500.

\section{III.3.1 HIPÓTESES (RESTRIÇÕES) UTILIZADAS NO MODELO}

As restrições têm a ver com os limites de potência que pode gerar cada motor (Eq. 2) e além disso com que a usina seja capaz de garantir a demanda mais as perdas (Eq.1).

\section{RESULTADOS E DISCUSSÕES}

Se qualquer das restrições não forem cumpridas, então o software desenvolvido usando os algoritmos genéticos emite as seguintes mensagens:

ERRO! A potência demandada é menor que a potência mínima. $\checkmark$ Potência Mínima: $0.56 \mathrm{~mW}>0.50 \mathrm{~mW}$ Potência Demandada.

Esta restrição está relacionada com a mínima capacidade da máquina de menor capacidade, garantindo que a capacidade demandada é maior do que a capacidade mínima de geração de uma das máquinas.

$\checkmark$ ERRO! Não é possível satisfazer a demanda com a capacidade atual.

$\checkmark$ Capacidade atual: $33.50 \mathrm{~mW}<200.00 \mathrm{~mW}$.

Esta restrição está relacionada com a capacidade máxima do conjunto de todas as máquinas, garantindo que a demanda de capacidade é menor que a capacidade máxima de geração da planta.

Os resultados depois de rodar o software foram os seguintes:

$\checkmark$ Despacho de Carga com Algoritmo Genéticos - SOLUÇÕES:

$\checkmark$ Demanda de Potência: $20 \mathrm{MW}$

$\checkmark$ Potência Mínima: $0.56 \mathrm{MW}$

$\checkmark$ Potência Máxima: $3.7 \mathrm{MW}$

$\checkmark$ Custo de Combustível: 1540.80 \$R

$\checkmark$ Perdas de Potência: 0.01 MW

Potência de cada Motor em MW:

\begin{tabular}{|c|c|c|c|c|c|c|c|c|c|c|}
\hline Pm1 & Pm2 & Pm3 & Pm4 & Pm5 & Pm6 & Pm7 & Pm8 & Pm9 & Pm10 & $\begin{array}{c}\text { P. } \\
\text { Total }\end{array}$ \\
\hline 3,34 & 3,70 & 3,60 & 3,35 & 3,42 & 0,00 & 0,00 & 0,84 & 0,00 & 1,76 & 20,01 \\
\hline
\end{tabular}

Fonte: Autores, (2107). mínima e máxima capacidade de potência das máquinas e os resultados do custo total de combustível, perdas totais de potência e potência ótima para cada uma das máquinas da planta para satisfazer a demanda de carga. Nas Figuras 1-5 são mostrados os gráficos mais importantes do algoritmo genético gerados em MATLAB.

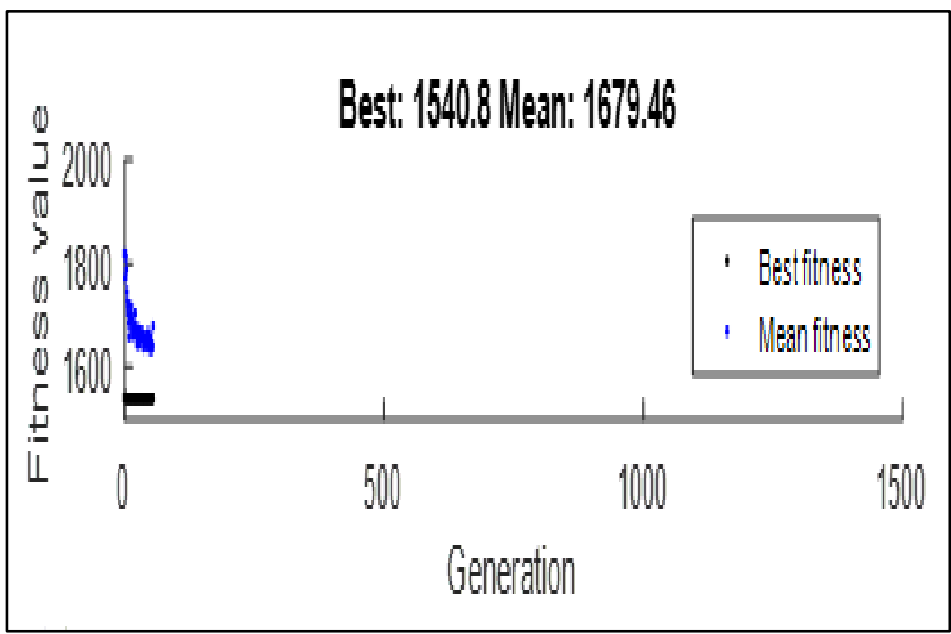

Figura 1: Valores da função atitude (fitness function) versus número de gerações.

Fonte: Autores, (2107).

Da figura 1 é possível ver que o melhor valor (best) do custo do consumo de combustível é 1540, que se corresponde com o mínimo custo. O gráfico também oferece o valor médio (mean) que é de 1679,46 reais.

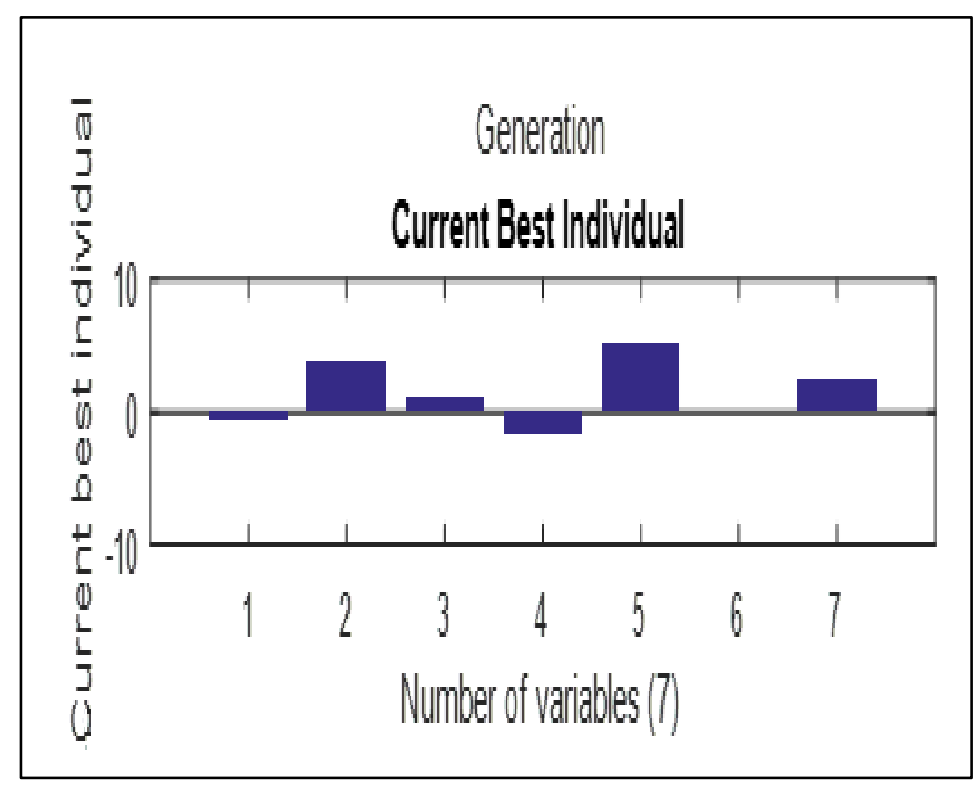

Figura 2: Melhor indivíduo de cada uma das variáveis. Fonte: Autores, (2107).

A figura 2 mostra o processo que efetua o algoritmo genético para ir procurando os valores das variáveis de tal forma que a função objetivo seja minimizada. 


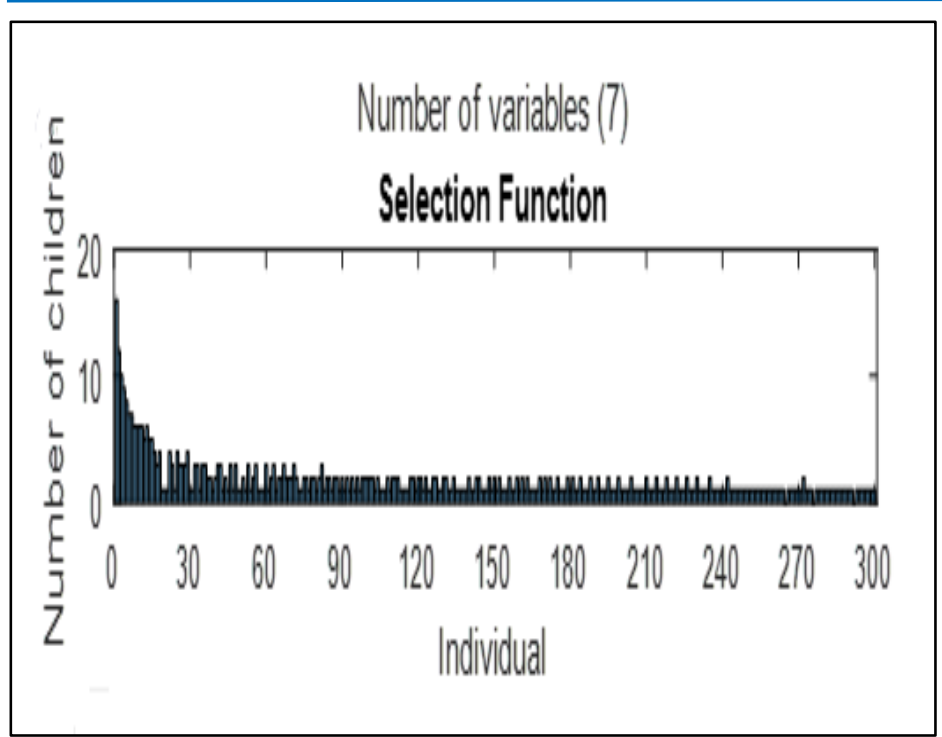

Figura 3. Número de descendentes para cada indivíduo durante a seleção dos resultados.

Fonte: Autores, (2107).

A figura 3 mostra o processo de mutação e cruzamento que permite ao algoritmo genético encontrar a melhor solução, se mostra o número de descendentes (children) e o número de indivíduos.

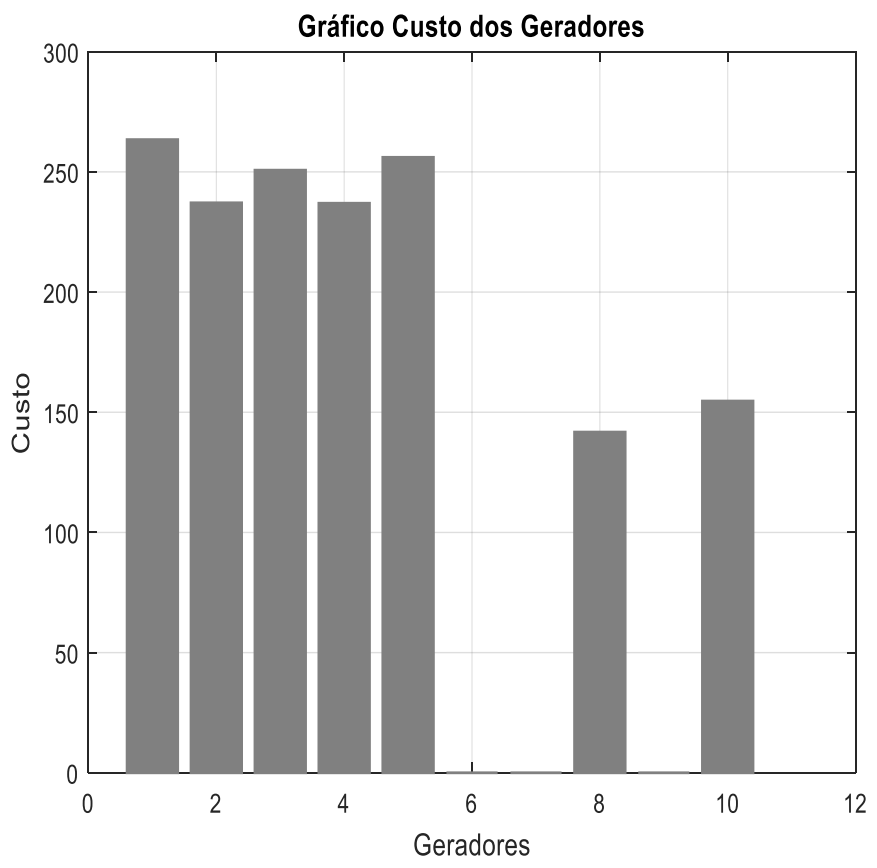

Figura 4: Custo de geração de cada gerador em reais.

Fonte: Autores, (2107).

O gráfico da figura 4 mostra o custo em que incorre cada gerador para atingir a demanda de potência dada. Do gráfico podese apreciar que os geradores 6, 7 e 9 estão desligados.

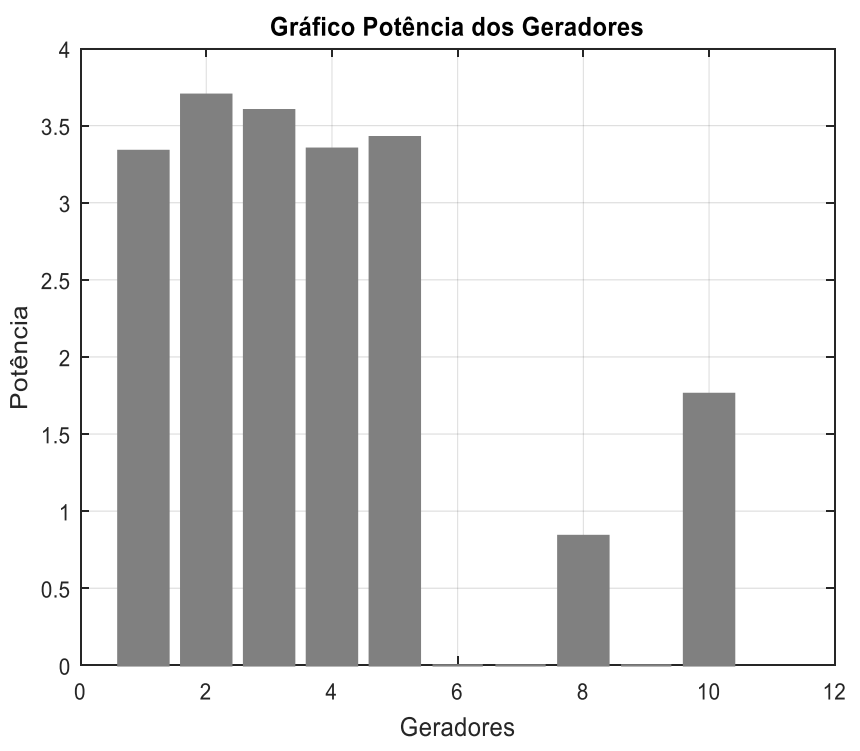

Figura 5. Potência Fornecida por cada gerador em MW. Fonte: Autores, (2017).

Da figura 5 observa-se a potência que está fornecendo cada gerador para atingir a demanda total. A desigualdade das potências dos motores selecionados tem muito a ver com os coeficientes de perda de cada motor, uma vez que o algoritmo seleciona os motores que têm menos perdas.

\section{CONCLUSÃO}

Este trabalho apresentou uma análise do problema do despacho econômico de carga e a solução do mesmo mediante os Algoritmos Genéticos. Para resolver o problema tem que ser oferecidos os dados de consumo de combustível e potência gerada por cada um dos motores além da demanda de potência desejada. Os métodos matemáticos convencionais, tais como o método de iteração lambda convergem rapidamente, mas a complexidade aumenta à medida que aumenta o tamanho do sistema.

Neste artigo o problema do despacho econômico de carga foi resolvido por o método de iteração lambda e o método de algoritmos genéticos, implementado em MATLAB. Foi analisado como estudo de caso uma usina de geração com 10 unidades ou motores. Os resultados obtidos estão de acordo como despacho de carga real. O método de iteração Lambda usando algoritmos genéticos é uma maneira simples de resolver o problema de despacho econômico de carga combons resultados

\section{REFERÊNCIAS}

[1] Fortunato, L. A. et al. Introdução ao planejamento da expansão e operação de sistemas de produção de energia elétrica. Niterói, Universidade Federal Fluminense, EDUFF, 1990.

[2] Aragón, V. S.; Esquivel, S. C.; Coello Coello, C. A. An immune algorithm with power redistribution for solving economic dispatch problems. Information Sciences, v. 295, n. 0, p. 609-632, 2/20/ 2015. ISSN 0020-0255. Disponível em: < http://www.sciencedirect.com/s cience/article/pii/S002002551401 $\underline{0081>\text {. }}$ 
[3] Wood, A. J.; Wollenberg, B. F. Power generation, operation, and control. John Wiley \& Sons, 2012. ISBN 111858595X.

[4] Reddy, J. M.; Kumar, N. D. Computational algorithms inspired by biological processes and evolution. Current Science (Bangalore), v. 103, n. 4, p. 370-380, 2012.

[5] barisal, A.; Prusty, R. Large scale economic dispatch of power systems using oppositional invasive weed optimization. Applied Soft Computing, v. 29, p. 122-137, 2015. ISSN 15684946.

[6] Michalewicz, Z.; Hartley, S. J. Genetic algorithms + data structures $=$ evolution programs. Mathematical Intelligencer, v. 18, n. 3, p. 71, 1996. ISSN 0343-6993.

[7] Holland, J. Holland, Adaptation in Natural and Artificial Systems: An Introductory Analysis with Applications to Biology, Control and Artificial Intelligence: MIT Press, Cambridge, MA 1992.

[8] Layec, A. ScicosLab: A Free Scientific Software Package. 1st HeDiSC Workshop, 2009.

[9] Lara, R. C. H. Herramientas de software libre para aplicaciones en ciencias e ingeniería. Revista Politécnica, v. 32, 2013. ISSN 1390-0129.

[10] Gilat, A. MATLAB com aplicações em Engenharia. Bookman Editora, 2009. ISBN 8540701871.

[11] Lages, E. N. Introdução ao MatLab. Universidade Federal de Alagoas-UFAL, Alagoas, 1999.

[12] Moore, H. MATLAB for Engineers. Prentice Hall Press, 2014. ISBN 0133485978.

[13] MarianI, V. C.; Preto, T. M.; Guedes, A. L. P. Utilização do Maple, MATLAB e SCILAB nos Cursos de Engenharia. COBENGE 2005-Congresso Brasileiro de Ensino de Engenharia, 2005.

[14] Sicupira, F. L. et al. Scilab versus Matlab. 2012.

[15] Park, J.-B. et al. A particle swarm optimization for economic dispatch with nonsmooth cost functions. IEEE Transactions on Power systems, v. 20, n. 1, p. 34-42, 2005. ISSN 0885-8950. 